\title{
Evaluation of the effects of applying the ventricular care bundle (VCB) method for reducing ventilator-associated pneumonia (VAP) in the intensive care unit of a general Chinese tertiary hospital
}

\author{
Weiping Liu, Yongfang Yang, Yueying Jiao, Kai Zhang, Yunting Hai, Haoxue Li, Huimin Xing, \\ Binbin Xu, Haibo Bai, Yuping Zhao, Huan Bao, Shuai Zhang, Wei Ren, Lifang Yang, Huijun Yang, \\ Junwei Tian, Meng Wang, Tianhui Guo \\ Department of Nosocomial Infection control, Inner Mongolia People's Hospital, Hohhot, China \\ Contributions: (I) Conception and design: W Liu, T Guo; (II) Administrative support: W Liu, Y Jiao, H Xing; (III) Provision of study materials or \\ patients: K Zhang, Y Hai, J Tian, H Yang; (IV) Collection and assembly of data: B Xu, H Bai, S Zhang, H Bao, M Wang, L Yang; (V) Data analysis \\ and interpretation: Y Zhao, Y Yang, H Li, W Ren; (VI) Manuscript writing: All authors; (VII) Final approval of manuscript: All authors. \\ Correspondence to: Tianhui Guo, MPH. Department of Nosocomial Infection Control, Inner Mongolia People's Hospital, No. 20 of Zhaowuda Street, \\ Saihan District, Hohhot 010017, China. Email: tianhui0409@126.com.
}

Background: Ventilator-associated pneumonia (VAP) is a severe complication that occurs within patients who must use ventilators in the intensive care unit (ICU). Ventilator care bundles (VCB) have been applied across many developed regions and have produced positive results in controlling VAP. In this study, we report on the implementation and effects of using VCBs to manage VAP in a general tertiary hospital in the Inner Mongolia Autonomous Region of China.

Methods: A targeted surveillance method was used to survey all the patients $(n=4,716)$ in the ICU from June 1, 2017 to May 31, 2019. Patients from June 1, 2017 to May 31, 2018, and June 1, 2018, to May 31, 2019, were respectively divided into 2 groups: the control group (2,029 patients) and intervention group (2,687 patients). These dates were selected because VCB was implemented from June 1, 2018, in our institution. The variables that were associated with VCB and observed were the head-of-bed elevation, oral care, maintenance of the pressure for the cuff of the endotracheal tube, aspiration of subglottic secretion, daily sedation vacation protocol, daily extubation assessment results, and hand hygiene. After collecting the data, the compliance of VCB, ventilator use ratio, and the incidence rate of VAP in these 2 groups were compared. Results: We observed that compliance with all of the intervention measures for VCB improved results in the intervention group compared to the control. Furthermore, the compliance rate of hand hygiene increased from $71.99 \%$ to $91.97 \%$, and the head-of-bed elevation of $30^{\circ}-45^{\circ}$ increased from $62.02 \%$ to $85.96 \%$. All differences between these two groups were statistically significant, according to the $\chi^{2}$-test. The ventilator use ratio was statistically and significantly lower in the intervention group (34.86\%) compared to the control group (40.29\%) $\left(\chi^{2}=95.513, \mathrm{P}<0.001\right)$. The incidence rate of VAP was statistically and significantly lower in the intervention group (13.70\%o) compared to the control group (18.85\%o) $\left(\chi^{2}=5.471, \mathrm{P}=0.019\right)$.

Conclusions: Our results show that VCB prevents VAP. Therefore, personnel training, clinical supervision, and surveillance feedback could promote a reduction in intervention measures.

Keywords: Bundle intervention; intensive care unit (ICU); incidence rate; ventilator-associated pneumonia (VAP)

Submitted Jan 28, 2020. Accepted for publication Jun 30, 2020.

doi: 10.21037/apm-20-289

View this article at: http://dx.doi.org/10.21037/apm-20-289 


\section{Introduction}

A ventilator is a commonly used medical device in the intensive care unit (ICU), and one of the most severe complications for a patient on a ventilator is ventilatorassociated pneumonia (VAP). VAP increases medical costs and extends the hospital length of stay (LOS) of ICU patients (1-3). While the length of ventilator use is a risk factor for VAP, the longer hospital LOS, patient age, and chronic disease are also risk factors (4). VAP is one of the most common types of healthcare-associated infections (HAI). Patients in the ICU usually have a significantly higher chance of having HAI than non-ICU patients. It is speculated that this is because most of them have severe conditions or decreased immune function. Among all of the HAI cases in the ICU, VAP accounts for $36-60 \%$ of all of cases (5). Furthermore, 9-27\% of patients on a ventilator will have VAP, and the infection rate of VAP is 1.2 8.5 cases per 1,000 days of ventilator use (6). Moreover, VAP infections can lengthen the LOS by 7-9 days (7).

VAP prevention interventions, such as oral care (5) and modified endotracheal tubes (8), could effectively reduce the chance of a VAP infection. Despite ICU patients only constituting $8 \%$ of all of the inpatients, a study conducted abroad revealed that $45 \%$ of all HAI occurred in the ICU (9). According to Vincent et al., 16\% of ICU patients will get infected with VAP (10). The death rate of VAP patients ranges from $24-76 \%$, which is $2-10$ times the death risk compared to non-VAP patients. As a result, VAP has become the main reason for HAI death (11). For this reason, reducing the prevalence of VAP in the ICU is a critical step for reducing HAI, improving the prognosis of patients on a ventilator in the ICU, and improving medical quality.

In 2001, the Institute for Healthcare Improvement (IHI) started to combine 4 VAP prevention interventions for ICU ventilator patients. Afterward, the ventilator bundle emerged in some studies conducted in developed countries. In 2005 Then the IHI formally published the ventilator care bundle (VCB) guidelines, which comprised 4 evidencebased VAP prevention interventions. These four methods included (I) elevating the head-of-bed by 30-45 degrees, (II) the conducting of daily sedation vacations, daily intubation and ventilation assessments, (III) peptic ulcer prevention, and (IV) deep venous thrombosis prevention (12). Bundle interventions can effectively prevent VAP infection, and these have already been broadly implemented worldwide, following the substantial results of a large number of previous studies (13-15). However, VCB has not been accepted by healthcare practitioners in the Inner Mongolia Autonomous Region, which is a border region of China. Inner Mongolia People's Hospital has required clinical healthcare practitioners to use VCB in all 6 ICUs since June 1, 2018. Our study is observational and compares the data from and before and after VCB implementation. We also concluded the value of implementing the bundle method.

In this study, 6 ICUs from different departments of a general tertiary hospital were selected for study to discuss the clinical value of the bundle intervention method in VAP prevention. The rates of interventions in the bundle before and after implementation were compared.

We present the following article in accordance with the STROBE Reporting Checklist (available at http://dx.doi. org/10.21037/apm-20-289).

\section{Methods}

\section{Surveillance objectives}

Surveillance was performed on all patients in the 6 ICUs (general ICU, cardiovascular medicine ICU, neurology ICU, respiratory ICU, neurosurgery ICU, and cardiovascular surgery ICU) of a general tertiary hospital in the Inner Mongolia Autonomous Region of China from June 1, 2017 to May 31, 2019. Patients from June 1, 2017 to May 31, 2018, were assigned as the control group, while patients from June 1, 2018 to May 31, 2019, were assigned as the intervention group. The study was approved by the Ethics Board of Inner Mongolia People's Hospital (No. 202000103L) and conducted in accordance with the Declaration of Helsinki. Since this study did not involving any patient's personal information and only medical record has been used in this study, informed consent is not required in this study.

\section{Surveillance method}

As a result of VCB not being widely accepted in the Inner Mongolia People's Hospital or the coverage regions, adverse medical care consequences for ventilator patients had occurred. Therefore, Inner Mongolia People's Hospital decided to require the clinical implementation of VCB since June 1, 2018. The implementation included the condition of requiring a medical quality assessment in the ICU department, which is managed by HAI practitioners.

The target surveillance method was used to perform a daily survey of all mechanical ventilator patients in the 
6 ICUs, according to the hospital-associated infection surveillance standards guidelines (16). A patient discharged from the ICU would be followed up for 48 hours after. All surveillance procedures were completed by the HAI practitioners from the Nosocomial Infection Department. They conducted an on-site observation of the performance conditions of the VAP intervention strategy in the ICU twice a week and recorded the findings. ICU nurses were responsible for filling in the ICU patient's daily record and the HAI case questionnaire based on the HAI patient's actual condition. The VAP diagnosis standard was based on the "VAP prevention, diagnosis and treatment guideline" published by the Intensive Care Branch of the Chinese Medical Association in 2013 (17).

\section{Intervention measure}

The VCB used in the present study was based on the "HAI prevention and control guidelines in ICU" (WS/T5092016) and other related evidence $(18,19)$. The VCB used in the present study included 8 interventions: head-of-bed elevation of 30-45 degrees, oral care with chlorhexidine 3-4 times per day, adherence to aseptic technique operations and hand hygiene guidelines, continuous evaluation of aspiration of subglottic secretion, maintenance of the cuff of the endotracheal tube with $25-30 \mathrm{~cm}$ of $\mathrm{H}_{2} \mathrm{O}$, the conducting of daily sedation vacation, and the conducting of daily intubation and ventilation use assessments.

\section{Evaluation indicator}

(I) The demographic information, including age, gender, chronic disease status, and surgical operations performed on the patient, in the intervention and control groups, were collected.

(II) Appendix D of the "Standard for nosocomial infection surveillance" published by the Ministry of Health (16) was used in the data collection strategy to calculate the patient's acute physiology and chronic health evaluation II (APACHEII) scores. Data on the ratio of ventilation use and incidence rate of VAP were also collected. The calculation equations are listed below:

* The ratio of use of ventilation = total number of ventilation use days/the total LOS of the patient $\times 100 \%$;

* The incidence rate of VAP = VAP infected patient number/the total number of ventilation use days $\times 100 \%$;
* Hand hygiene compliance $=$ actual times of hand hygiene performance/supposed times of hand hygiene performance $\times 100 \%$;

* Performance ratio of a head-of-bed elevation of $30^{\circ}=$ person-days of a head-of-bed elevation of $30 \%$ the total number of ventilation use days $\times 100 \%$;

- Performance ratio of oral care $>3$ times $=$ personday of oral care $>3$ times per day/total number of ventilation use days $\times 100 \%$;

* Performance ratio of continuous aspiration of subglottic secretion $=$ person-days of continuous aspiration of subglottic secretion/the total number of ventilation-use days $\times 100 \%$;

* Performance ratio of extubation assessment = person-days of extubation assessment/the total number of ventilation-use days $\times 100 \%$.

\section{Statistical analysis}

SPSS 19.0 software was used to conduct the statistical analysis, while an $\chi^{2}$-test was used to compare the enumeration data between the 2 groups. A P value $<0.05$ was considered statistically significant.

\section{Results}

\section{Demographic information}

Four thousand seven hundred and sixteen patients underwent surveillance. Two thousand six hundred and eighty-seven patients were assigned to the intervention group, and 2,029 patients were assigned to the control group. The differences in gender, age, chronic disease, and surgical operation of patients in these two groups were not statistically significant $(\mathrm{P}>0.05)$; therefore, these two groups are comparable (Table 1).

\section{Compliance of $V C B$}

Hand hygiene compliance, the performance ratio of headof-bed elevation of 30-45 degrees, performance ratio of oral care $>3$ times per day, the performance ratio of adhering to the management of the cuff of the endotracheal tube, the performance ratio of continuous aspiration of subglottic secretion measurement, the performance ratio of undergoing sedation vacation, and the performance ratio of extubation assessment all increased after the VCB was 
Table 1 Demographic information of the patient in two groups

\begin{tabular}{|c|c|c|c|c|}
\hline Demographic information & Intervention group & Control group & $\chi^{2}$ & $\mathrm{P}$ \\
\hline Male & 1,436 & 1,047 & 1.571 & 0.210 \\
\hline Female & 1,251 & 982 & & \\
\hline \multicolumn{5}{|l|}{ Age (years old) } \\
\hline$\leq 65$ & 1,225 & 891 & & \\
\hline \multicolumn{5}{|l|}{ Chronic disease } \\
\hline Yes & 1,447 & 1,122 & 0.975 & 0.323 \\
\hline No & 1,240 & 907 & & \\
\hline No & 1,309 & 1,010 & & \\
\hline
\end{tabular}

Table 2 Compliance comparison of before and after VCB implementation

\begin{tabular}{|c|c|c|c|c|}
\hline Measure & Intervention group, $\mathrm{n}(\%)$ & Control group, n (\%) & $\chi^{2}$ & $P$ \\
\hline Head-of-bed elevation & $1,148 / 1,851(62.02)$ & 1,231/1,432 (85.96) & 231.964 & $<0.001$ \\
\hline Oral care & $1,238 / 1,876(65.99)$ & $1,232 / 1,450(84.97)$ & 154.054 & $<0.001$ \\
\hline Maintain endotracheal tube cuff pressure & $1,088 / 1,860(58.49)$ & 1,225/1,442 (84.95) & 270.994 & $<0.001$ \\
\hline Sedation vacation & $1,243 / 1,866(66.61)$ & $1,350 / 1,444(93.49)$ & 151.735 & $<0.001$ \\
\hline Extubation assessment & $1,344 / 1,868(71.95)$ & 1,343/1,445 (92.94) & 234.291 & $<0.001$ \\
\hline
\end{tabular}

VCB, ventricular care bundle.

implemented. All differences between the two groups were statistically significant $(\mathrm{P}<0.001)$, according to the $\chi^{2}$-test (Table 2).

\section{Ventilation use and VAP infection}

The ventilation use ratio in the intervention group was $34.86 \%$, which was statistically lower than in the control group $(40.29 \%)\left(\chi^{2}=95.513, \mathrm{P}<0.001\right)$. Furthermore, the incidence rate of VAP in the intervention group was $13.70 \%$, which was statistically lower than in the control group $(18.85 \%)\left(\chi^{2}=5.471, \mathrm{P}=0.019\right.$; Table 3).

\section{Discussion}

\section{VAP prevention and control measures}

The VCB method is a collection of evidence-based interventions that focus on ventilator patient care and treatment. The VCB used in the present study was based on the detailed guidelines set domestically and internationally that factored in the variables of the study's hospitals. Improving the prognosis and reducing the incidence rate of VAP is the goal of VCB. The surveillance of the VCB implementation process and increasing the compliance to VCB was achieved during its implementation. A study 
Table 3 Before and after intervention ventilation use and VAP infection rates

\begin{tabular}{lccccc}
\hline Group/statistics & Surveillance case & LOS (stays) & Ventilation use days & Ventilation use ratio (\%) & VAP incidence rate (\%, case) \\
\hline Intervention group & 2,687 & 1,8838 & 6,567 & 34.86 & $13.70[90]$ \\
Control group & 2,029 & 12,598 & 5,076 & 40.29 & $18.85[98]$ \\
$\chi^{2}$ & - & - & - & 95.513 & 5.471 \\
$\mathrm{P}$ & - & - & - & $<0.001$ & 0.019 \\
\hline
\end{tabular}

VAP, ventilator-associated pneumonia; LOS, length of stay.

revealed that compliance to VAP prevention compared to the control, bundle intervention strategy increased the results from $82.06 \%$ in 2012 to $96.88 \%$ in 2013 , and the VAP infection rate decreased from $32.72 \%$ in 2012 to $24.60 \%$ in 2013 (20). In this study, the fulltime professionals from the Nosocomial Management Department, the director of the ICU, and the head nurse supervised compliance to VCB implementation. Feedback on its implementation was communicated to all healthcare workers (HCWs) of the ICU Department to improve the VCB implementation conditions. As a result, the compliance rate of all measures significantly increased after this implementation strategy.

Hand hygiene is always accompanied by other measures for preventing and controlling VAP. According to a study on basic prevention measures, including hand hygiene, in eight pediatric intensive care unit (PICUs) in five developing countries, the compliance of hand hygiene increased from $48.9 \%$ to $67.1 \%$ after implementing a series of prevention measures. As a result of this implementation, the incidence rate of VAP decreased from 11.7 per 1,000 ventilation use days to 8.1 per 1,000 ventilation use days (13). The increased compliance of hand hygiene is beneficial for reducing the incidence rate of VAP. As well as being an important variable in HAI prevention, hand hygiene is also a vital variable in VAP.

The patient's body position is also an important variable for VAP infection. A prospective study on VAP prevention from 1966 to 2001 revealed that the half supine position is the best position for VAP prevention. The evidence for this is strong, and all ventilator patients should choose the half supine position when there are no contraindications (21). These guidelines require that the cephalothorax of the patient should be elevated at $30^{\circ}-45^{\circ}$ when there are no contraindications. During the present implementation period, the HCWs found a convenient method for easily meeting this elevation requirement. The method was done by conspicuously marking the $30^{\circ}$ and $45^{\circ}$ elevation position on one side of the head-of-bed previously measured by a protractor. The HCWs elevated the head-of-bed between two marks on the side of the bed, achieving an elevation angle within $30^{\circ}-45^{\circ}$. Through this approach, HCWs do not need to measure the elevation angle every single time the head needs to be elevated. The mark that was previously made could also help other HCWs easily find the right angle of the head-of-bed elevation quickly. The reasons for the low compliance of head-of-bed elevation included a lack of awareness of the benefits of the half supine position, the lack of execution responsibility, and the lack of practice feasibility and ignorance of this strategy. As for the nurses, they concluded that the doctors did not provide clear enough instructions for adjusting the head-of-bed elevation, which is the main reason for the low compliance since nurses always adhere strictly to the instructions of the doctor. Therefore, doctors should provide clear instructions for setting the head-of-bed elevation during the execution of this strategy. Although the importance of the head-of-bed elevation being constantly pushed during the complement period, the compliance rates were still not high since this intervention burdened the workload of the nurses, in which the half supine position of the patient required more care work than the supine position. Therefore, the nurses on duty should all take responsibility for executing the head-of-bed elevation and reprimand non-executing nurses for ensuring compliance.

The establishment of artificial airways breaks the oral and nasal vessel's natural barriers against bacteria. Hence, strict, and effective oral care in ventilation is needed for the patient to protect their airway. Substantial evidence has revealed that comprehensive oral care, a part of the $\mathrm{VCB}$, could reduce the incidence rate of VAP. A meta-analysis of 2,341 adult patients across 12 studies using chlorhexidine to prevent VAP in their oral care procedure had a reduced risk of VAP (22). Oral care $>3$ times per day using chlorhexidine was required in the present study. Chlorhexidine has low 
irritation and strong broad-spectrum bactericidal effects, which is effective against gram-negative and positive bacteria. Furthermore, chlorhexidine has a positive charge, which can absorb the negative charge in dental plaque and other bacteria in the oral mucosa surface during oral care procedure producing a gradual and persistent anti-bacterial effect.

Aspiration prevention is also an important strategy for preventing VAP. Presently, the maintenance of endotracheal tube cuff pressure is a widely used clinical prevention strategy. According to the "Practical guidelines for mechanical ventilation published by the Society of Critical Care Medicine, Chinese Medical Association" in 2006 (23), the pressure of the artificial airway of the endotracheal tube should undergo routine surveillance. The pressure should be maintained between 25 to $30 \mathrm{cmH}_{2} \mathrm{O}$, and high-volume low-pressure should be maintained. These would enclose not only the airway, but it would simultaneously also maintain a pressure that is not higher than the perfusion pressure of the airway mucous blood capillary. Furthermore, this could also prevent airway mucosa ischemic injury, trachea-esophageal fistula, tracheostenosis after extubation, and other complications. It has been demonstrated that under-inflation of the balloon and low pressure cannot effectively enclose the space between the balloon and airway, leading to secreta above the balloon. The secreta would enter the lower respiratory tract and increase the risk of a pneumonia infection. Another study revealed that compared to staggered surveillance, constant surveillance of the endotracheal cuff pressure and maintaining it at $25 \mathrm{cmH}_{2} \mathrm{O}$ could effectively reduce the incidence rate of VAP (24). Equipping an endotracheal pressure detector in the ICU and having constant surveillance allowed for the compliance of endotracheal cuff pressure to increase from $65.99 \%$ to $84.97 \%$.

For patients using an indwelling ventilator catheter, secreta in the upper respiratory tract could gather above the balloon of the ventilator catheter leading to local bacteria reproduction. Then, secreta could follow the respiratory tract and enters the lungs, causing a pulmonary infection. Therefore, aspiration of subglottic secretion could effectively reduce pulmonary infection rates (25-27). Another study showed that constant subglottic secretion aspiration could reduce the incidence rate of VAP, shorten the length of ventilation use time, shorten the LOS while having no obvious adverse reaction s, and good patient tolerance (28).

Constant intravenous injection sedation could increase ventilation use time and LOS. Therefore, a sedation vacation is recommended, including the VCB for VAP prevention. A clinical randomized control trial of 128 patients receiving mechanical ventilation in ICU showed that the implementation of awakening the patients daily as a sedation vacation in the morning and extubation every day resulted in the reduction of the average ventilation use time from 7.3 to 4.9 days $(\mathrm{P}=0.004)$ and shortened the LOS from 9.9 to 4.9 days $(\mathrm{P}=0.02)$. The incidence rate of VAP and medical costs was also reduced due to daily awakening (29). Sedation vacation is useful in preventing VAP and is a necessary component of the VCB.

Invasive ventilation needs to open the airway resulting in a higher chance of pathogen bacteria being introduced to the lower respiratory tract. Prolonged ventilation use time also increases the risk of lower respiratory tract infections that causes a VAP infection. A study showed that the chance of VAP infection increased with longer ventilation use time. Further, it has been demonstrated that ventilation use time is an independent risk factor in VAP infection (30). The reason for this could be longer ventilation use time, longer airway open time, more invasive of operation, a higher chance of infection by a contaminated ventilator, and a higher risk of getting ventilation related complications. Therefore, the doctor should assess daily the necessity of ventilation and intubation use. Extubation should be performed as soon as the patient meets the extubation requirement to reduce ventilation use time.

\section{Obvious effects of VCB in preventing VAP}

VCB is a group of intervention measures based on evidencebased guidelines. The implementation of these strategies altogether has better VAP prevention effects compared to only single intervention measure implementation. An American multi-center cohort study of mechanical ventilation bundle intervention measures showed that compliance with the bundle techniques increased from $32 \%$ to $84 \%$ and the overall incidence rate of VAP reduced from $5.5 \%$ to $0.0 \%$ after 30 months. These techniques included head-of-bed elevation, stress ulcer prevention, deep venous thrombosis prevention, sedation vacation, and extubation assessment (15). The choice of using all interventions of the VCB in this study is based not only on literature evidence but also on real clinical situations. The head-of-bed elevation, oral care techniques, maintaining endotracheal tube cuff pressure, the aspiration of subglottic secretion, daily sedation vacation, daily extubation assessment, and 
hand hygiene supervision surveillance were chosen in our VCB. After one year of implementation, the incidence rate of VAP and the ventilator-used ratio had a significant reduction compared to before implementation showing that VCB in this study has a significant effect of reducing VAP infection in ICU.

\section{VCB promotion by personnel training and supervision feedback}

VCB is a new strategy for VAP prevention and control, and it could significantly reduce the incidence rate of VAP based on the existing evidence. However, HCW's compliance in using VCB methods should be paid more attention to enhancing the effectiveness of every step in achieving prevention purposes (31). Therefore, training the related HCWs before implementation is suggested to educate them on the importance of VCB and to implement it better. Detailed quality control standards should be made before implementation, and efficient managers should be in charge as the director of the department and as the chief nurse. The Director of the department and chief nurse should regularly evaluate the situation, gain valuable feedback, and improve the implementation as needed (32). During the VCB implementation period, a supervision evaluation chart should be used to record the implementation and the conditions of every intervention measure for achieving explicit purposes and significant effects. Continued surveillance and feedback could also significantly improve HCW's awareness and help them to execute HAI prevention and control methods better. A Chinese study investigated VAP patients in general ICUs when compliance with VCB increased, mechanical ventilation use days decreased after training the ICU HCW (33). In this study, clinical HCW received HAI surveillance data every month and compared the reported data from home and abroad, which let HCW quantize the effect of prevention and control, and see the difference in real-time. These reports give the HCWs a direct understanding of VAP's urgent status in their department and to further enhance awareness for prevention and control.

\section{Conclusions}

In conclusion, head-of-bed elevation, oral care, maintaining endotracheal tube cuff pressure, aspiration of subglottic secretion, daily sedation vacation, daily extubation assessment, hand hygiene, and supplemented surveillance feedback and supervision of the VCB implementation could effectively reduce the incidence rate of VAP in our studied hospital. However, this study is only limited to one hospital. Since every hospital has different basic measures of VAP prevention and control in their ICU, extrapolating this result in larger scenarios needs further research.

\section{Acknowledgments}

Funding: This work is supported by Department of Human Resources and Social Security of Inner Mongolia; State Key Laboratory for Infectious Disease Prevention and Control of Chinese Center for Disease Control and Prevention (No. 2019SKLID305); Department of Science \& Technology of Inner Mongolia [No. 2017MS(LH)0845]; Infection Prevention and Control Research Fund Administration Commission of China Geriatric Society (No. GRYJLRK2018021); and Health Commission of Inner Mongolia (No. 201703006).

\section{Footnote}

Reporting Checklist: The authors have completed the STROBE Reporting Checklist. Available at http://dx.doi. org/10.21037/apm-20-289

Data Sharing Statement: Available at http://dx.doi. org/10.21037/apm-20-289

Conflicts of Interest: All authors have completed the ICMJE uniform disclosure form (available at http://dx.doi. org/10.21037/apm-20-289). Dr. WL, Dr. YY, Dr. YJ, Dr. KZ, Dr. YH, Dr. HL, Dr. HX, Dr. BX, Dr. HB, Dr. YZ, Dr. TG report grants from Department of Science \& Technology of Inner Mongolia, grants from Department of Human Resources and Social Security of Inner Mongolia, grants from State Key Laboratory for Infectious Disease Prevention and Control of Chinese Center for Disease Control and Prevention, grants from Infection Prevention and Control Research Fund Administration Commission of China Geriatric Society, grants from Health Commission of Inner Mongolia, during the conduct of the study. The other authors have no conflicts of interest to declare.

Ethical Statement: The authors are accountable for all aspects of the work in ensuring that questions related to the accuracy or integrity of any part of the work are appropriately investigated and resolved. The study was 
approved by the Ethics Board of Inner Mongolia People's Hospital (No. 202000103L) and conducted in accordance with the Declaration of Helsinki. Since this study did not involving any patient's personal information and only medical record has been used in this study, informed consent is not required in this study.

Open Access Statement: This is an Open Access article distributed in accordance with the Creative Commons Attribution-NonCommercial-NoDerivs 4.0 International License (CC BY-NC-ND 4.0), which permits the noncommercial replication and distribution of the article with the strict proviso that no changes or edits are made and the original work is properly cited (including links to both the formal publication through the relevant DOI and the license). See: https://creativecommons.org/licenses/by-nc-nd/4.0/.

\section{References}

1. Barnett AG, Beyersmann J, Allignol A, et al. The timedependent bias and its effect on extra length of stay due to nosocomial infection. Value Health 2011;14:381-6.

2. Rosenthal VD, Udwadia FE, Muñoz HJ, et al. Timedependent analysis of extra length of stay and mortality due to ventilator-associated pneumonia in intensive-care units of ten limited-resources countries: findings of the International Nosocomial Infection Control Consortium (INICC). Epidemiol Infect 2011;139:1757-63.

3. Rosenthal VD, Maki DG, Jamulitrat S, et al. International Nosocomial Infection Control Consortium (INICC) report, data summary for 2003-2008, issued June 2009. Am J Infect Control 2010;38:95-104.e2.

4. American Thoracic Society; Infectious Diseases Society of America. Guidelines for the management of adults with hospital-acquired, ventilator-associated, and healthcareassociated pneumonia. Am J Respir Crit Care Med 2005;171:388-416.

5. Gupta A, Gupta A, Singh TK, et al. Role of oral care to prevent VAP in mechanically ventilated Intensive Care Unit patients. Saudi J Anaesth 2016;10:95-7.

6. Skrupky L P, McConnell K, Dallas J, et al. A comparison of ventilator-associated pneumonia rates as identified according to the National Healthcare Safety Network and American College of Chest Physicians criteria. Crit Care Med 2012;40:281-4.

7. Afshari A, Pagani L, Harbarth S. Year in review 2011: Critical Care-infection. Crit Care 2012;16:242.

8. Deem S, Yanez D, Sissons-Ross L, et al. Randomized pilot trial of two modified endotracheal tubes to prevent ventilator-associated pneumonia. Ann Am Thorac Soc 2016;13:72-80.

9. Donowitz LG, Wenzel RP, Hoyt JW. High risk of hospital-acquired infection in the ICU patient. Crit Care Med 1982;10:355-7.

10. Vincent JL, Bihari DJ, Suter PM, et al. The prevalence of nosocomial infection in intensive care units in Europe. Results of the European Prevalence of Infection in Intensive Care (EPIC) Study. EPIC International Advisory Committee. JAMA 1995;274:639-44.

11. Cavalcanti M, Valencia M, Torres A. Respiratory nosocomial infections in the medical intensive care unit. Microbes Infect 2005;7:292-301.

12. Berwick DM, Calkins DR, Mccannon CJ, et al. The 100 000 Lives Campaign: Setting a Goal and a Deadline for Improving Health Care Quality. JAMA 2006;295:324-7.

13. Rosenthal VD, Álvarez-Moreno C, Villamil-Gómez W, et al. Effectiveness of a multidimensional approach to reduce ventilator-associated pneumonia in pediatric intensive care units of 5 developing countries: International Nosocomial Infection Control Consortium findings. Am J Infect Control 2012;40:497-501.

14. Li Y, Xu W, Ge W, et al. Impact of integrated intervention measures on the compliance of ventilator care bundles by medical staff and the incidence of ventilator associated pneumonia of patients in general intensive care unit. Chinese Journal of Nosocomiology 2017;27:1751-3.

15. Berenholtz SM, Pham JC, Thompson DA, et al. Collaborative cohort study of an intervention to reduce ventilator-associated pneumonia in the intensive care unit. Infect Control Hosp Epidemiol 2011;32:305-14.

16. WS/T 312-2009, Standard for nosocomial infection surveillance. Beijing: Ministry of Health of the People's Republic of China, 2009.

17. Intensive Care Branch of Chinese Medical Association. Guideline for the prevention, diagnosis and treatment of ventilator-associated pneumonia (2013). Chinese Journal of Internal Medicine 2013;52:524-543.

18. Venkatram S, Rachmale S, Kanna B. Study of device use adjusted rates in health care-associated infections after implementation of "bundles" in a closed-model medical intensive care unit. J Crit Care 2010;25:174.e11-174.e18.

19. Zheng W, Mao Y, Zhou H, et al. Effect of bundle intervention on ventilator-associated pneumonia in an intensive care unit. Chinese Journal of Infection Control 2016;15:31-3.

20. Gao F, Wu YY, Zou JN, et al. Impact of a bundle on 
prevention and control of healthcare associated infections in intensive care unit. Journal of Huazhong University of Science and Technology [Medical Sciences] 2015;35:283-90.

21. Collard, Harold R. Prevention of Ventilator-Associated Pneumonia: An Evidence-Based Systematic Review. Ann Intern Med 2003;138:494-501.

22. Labeau SO, Vyver K, Brusselaers N, et al. Prevention of ventilator-associated pneumonia with oral antiseptics: A systematic review and meta-analysis. Lancet 2011;11:845-54.

23. Practical guidelines for mechanical ventilation (2006). Beijing: Society of Critical Care Medicine, Chinese Medical Association, 2006.

24. Nseir S, Zerimech F, Fournier C, et al. Continuous control of tracheal cuff pressure and microaspiration of gastric contents in critically ill patients. Am J Respir Crit Care Med 2011;184:1041-7.

25. Leasure AR, Stirlen J, Lu SH. Prevention of ventilatorassociated pneumonia through aspiration of subglottic secretions: a systematic review and meta-analysis. Dimens Crit Care Nurs 2012;31:102-17.

26. Wang F, Bo L, Tang L, et al. Subglottic secretion drainage for preventing ventilator-associated pneumonia: an updated meta-analysis of randomized controlled trials. J Trauma Acute Care Surg 2012;72:1276-85.

27. Liu H, Xiao P, Li J, et al. For CASS in prevention of VAP effect research. China Health Standard Management

Cite this article as: Liu W, Yang Y, Jiao Y, Zhang K, Hai Y, Li H, Xing H, Xu B, Bai H, Zhao Y, Bao H, Zhang S, Ren W, Yang L, Yang H, Tian J, Wang M, Guo T. Evaluation of the effects of applying the ventricular care bundle (VCB) method for reducing ventilator-associated pneumonia (VAP) in the intensive care unit of a general Chinese tertiary hospital. Ann Palliat Med 2020;9(5):2853-2861. doi: 10.21037/apm-20-289
2015;6:95-6.

28. Xia L, Li M, Chen L. Apply of tracheotomy catheter aspiration of subglottic secretion in ICU. Anhui Medical Journal 2014;35:1165-6.

29. Kress J P, Pohlman A S, Michael F, et al. Daily interruption of sedative infusions in critically ill patients undergoing mechanical ventilation. N Engl J Med 2000;342:1471-7.

30. American Thoracic Society; Infectious Diseases Society of America. Guidelines for the management of adults with hospital-acquired, ventilator-associated, and healthcareassociated pneumonia. Am J Respir Crit Care Med 2005;171:388-416.

31. Berenholtz SM, Pham JC, Thompson DA, et al. Collaborative cohort study of an intervention to reduce ventilator-associated pneumonia in the intensive care unit. Infect Control Hosp Epidemiol 2011;32:305-14.

32. Zhu J. Value analysis of bundle intervention and prevention of ventilator-associated pneumonia. China Modern Medicine 2014;21:105-7.

33. Shan J, Wu J, Gu Y, et al. The clinical study of cluster intervention strategies for the prevention of ventilator associated pneumonia. Journal of Nurses Training, 2012;27:202-5.

34. Pu D, Tang $\mathrm{H}$, Zong $\mathrm{Z}$, et al. Effect of staff training on compliance of bundle to prevent ventilator-associated pneumonia. Chinese Journal of Nosocomiology 2013,23:1545-7. 